\title{
CORRECTION
}

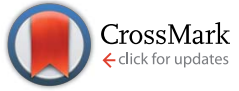

Cite this: J. Mater. Chem. A, 2015, 3, 16874

\section{Correction: A low cost azomethine-based hole transporting material for perovskite photovoltaics}

\author{
M. L. Petrus, ${ }^{a b}$ T. Bein, $^{a}{ }^{\text {T. J. Dingemans }}{ }^{\star b}$ and P. Docampo*a
}

DOI: $10.1039 / c 5 \operatorname{ta} 90163 d$

www.rsc.org/MaterialsA

Correction for 'A low cost azomethine-based hole transporting material for perovskite photovoltaics' by M. L. Petrus et al., J. Mater. Chem. A, 2015, 3, 12159-12162.

The Acknowledgements section was omitted from the above manuscript. Please find the Acknowledgments below.

\section{Acknowledgements}

The authors acknowledge funding from the Bavarian Ministry of the Environment and Consumer Protection, the Bavarian Network "Solar Technologies Go Hybrid", and the DFG Excellence Cluster Nanosystems Initiative Munich (NIM). P. D. acknowledges support from the European Union through the award of a Marie Curie Intra-European Fellowship. Furthermore, the authors want to thank Andreas Binek for performing the SEM experiments.

The Royal Society of Chemistry apologises for these errors and any consequent inconvenience to authors and readers. 